\title{
Core Entrustable Professional Activities for Entering Residency Pilot Group Update: Considerations for Medical Science Educators
}

\author{
Kimberly D. Lomis ${ }^{1} \cdot$ Michael S. Ryan ${ }^{2} \cdot$ Jonathan M. Amiel $^{3} \cdot$ Patrick M. Cocks $^{4}$. \\ Margaret O. Uthman $^{5} \cdot$ Karin F. Esposito ${ }^{6}$
}

Published online: 6 July 2016

(C) International Association of Medical Science Educators 2016

\section{Introduction}

One important goal of undergraduate medical education (UME) is to prepare students for their clinical duties as interns. Increasingly, program directors in graduate medical education (GME) have noted significant variability in medical school graduates' readiness to perform routine clinical activities, and this may affect patient care during July transitions [1]. In response, the Association of American Medical Colleges (AAMC) convened an experienced panel, including a student, a resident, a basic scientist, and clinical educators, to identify the most important activities that interns should be able to perform without direct supervision on the first day of residency. A national reactor panel of medical educators provided iterative feedback. In May 2014, the AAMC published a cur-

Kimberly D. Lomis

kim.lomis@vanderbilt.edu

1 Office of Undergraduate Medical Education, Vanderbilt University School of Medicine, 201 Light Hall, Nashville, TN 37232, USA

2 Clinical Medical Education, Virginia Commonwealth University, 1201 E. Marshall St, Richmond, VA 23220, USA

3 Office of Education, Columbia University College of Physicians \& Surgeons, 630 West 168th Street, Room 3-401, New York, NY 10032, USA

4 NYU School of Medicine, Bellevue Hospital Center, 550 First Avenue, Room NBV-16N30, New York, NY 10016, USA

5 University of Texas McGovern Medical School, 6431 Fannin St, Houston, TX 77030, USA

6 Herbert Wertheim College of Medicine, Florida International University, 11200 SW 8th Street, AHCII 662, Miami, FL 33199 , USA riculum developer's guide, entitled Core Entrustable Professional Activities for Entering Residency [2] (Table 1).

Entrustable professional activities (EPAs) describe units of work that represent routine activities of physicians, as distinguished from competencies, which describe observable abilities of an individual [3]. Both constructs are important to fostering the development of trainees. Successful performance of an EPA requires that the trainee synthesize multiple competencies in a cohesive manner in order to care for a patient. If the trainee performs the task in a satisfactory manner, it can be inferred that the trainee possesses the associated competencies. If, however, the performance is not satisfactory, it is helpful to refer back to the associated competencies to provide clear feedback and develop a plan for improvement. EPAs together constitute the core activities of the professional [3].

The term entrustment is intentional and should carry significant implications for both assessors and learners. The construct of entrustment dispels the notion that it is sufficient to simply possess knowledge, skills, and attitudes. Ultimately, supervisors must trust the learner to perform relevant tasks in the care of patients, without direct supervision.

Clinical supervisors make implicit, ad hoc, entrustment decisions every day [4]. The intent of the EPA framework is to capitalize upon this intuitive clinical perspective on performance. To prepare students to succeed in this competencybased framework, it will be necessary not only to teach and assess knowledge, skills, and attitudes relevant to EPAs throughout the UME curriculum; it is equally critical to promote and monitor the development of key attributes that engender trust. Formal entrustment decisions using multiple sources of evidence can ensure that each individual graduate is prepared for internship.

In an effort to provide guidance to the medical education community regarding the implementation of the EPA framework in undergraduate medical education, the AAMC 
Table 1 The 13 Core Entrustable Professional Activities for Entering Residency

1. Gather a history and perform a physical examination

2. Prioritize a differential diagnosis following a clinical encounter

3. Recommend and interpret common diagnostic and screening tests

4. Enter and discuss orders and prescriptions

5. Document a clinical encounter in the patient record

6. Provide an oral presentation of a clinical encounter

7. Form clinical questions and retrieve evidence to advance patient care

8. Give or receive a patient handover to transition care responsibility

9. Collaborate as a member of an inter-professional team

10. Recognize a patient requiring urgent or emergent care and initiate evaluation and management

11. Obtain informed consent for tests and/or procedures

12. Perform general procedures of a physician

13. Identify system failures and contribute to a culture of safety and improvement

initiated a pilot. Teams from ten schools (Table 2) were convened to explore how to promote readiness for entrustment by the time students enter residency. The pilot group has reviewed literature, engaged in active discussions, and initiated development of tools for teaching and assessments. After a year of deliberation, this group generated a list of guiding principles for UME institutions planning to enact the EPA framework [5]. This commentary represents the current thinking of the Steering Committee for the Core EPA Pilot regarding the implications of EPAs for medical science educators.

\section{Medical Science Educators and EPAs in the Pre-Clerkship Phase of Medical Training}

Entrustment is fundamentally a workplace construct. However, successful performance of EPAs in residency relies upon a developmental process that begins early in medical training and requires experiences that are vertically integrated throughout the curriculum. Many of the core EPAs can be described as a collection of smaller components, or "stepping stones," that build to the ultimate task. Some of these components must be introduced and practiced at early stages of training, before the student is ready to participate in the direct care of patients [6].

Table 2 The 10 Core EPA Pilot Schools

Columbia University College of Physicians and Surgeons

Florida International University Herbert Wertheim College of Medicine

McGovern Medical School at The University of Texas Health Science

Center at Houston (UTHealth)

Michigan State University College of Human Medicine

New York University School of Medicine

Oregon Health \& Science University School of Medicine

University of Illinois College of Medicine

Vanderbilt University School of Medicine

Virginia Commonwealth University School of Medicine

Yale School of Medicine
The Curriculum and Assessment Concept Group for the Core EPA Pilot recommends a system-based approach to the incorporation of content and assessments relevant to EPAs throughout each institution's curriculum. As integral members of curricular planning teams, medical science educators should consider components relevant to EPAs that can be introduced early. Familiarity with the Core EPAs will enhance the incorporation of appropriate content (Table 3).

The Core EPA Curricular Developer's Guide offers a rich description of each EPA, describing key functions, associated competencies, and expected behaviors [2]. Additionally, vignettes provide insight into how these components come together in patient scenarios to demonstrate entrustable levels of performance. The pilot group is in the process of describing additional levels of performance leading up to the entrustable expectations. This will allow early educators to design curriculum appropriate to learners' needs and to monitor whether learners are on a trajectory for success in their clinical work.

Medical science educators have extensive experience fostering the process of maturation that many entering students undergo in the transition from college to medical school. As stated in the AAMC Curriculum Developer's Guide, "two competencies are foundational to all of the EPAs because they are required for any entrustment decision: 1) trustworthiness and 2) self-awareness of limitations that leads to appropriate help-seeking behavior" [2]. Trustworthiness and self-awareness should be emphasized from day one of medical school.

The Entrustment Concept Group for the Core EPA Pilot has been exploring the prospect of explicitly measuring trustworthiness in learners. Fundamental to entrustment, trustworthiness has been described by Kennedy et al. [7] as encompassing three key aspects: truthfulness, conscientiousness, and discernment (awareness of one's limitations). The trend in pre-clerkship educational programs to incorporate more active and team learning formats enhances the ability of teachers in the pre-clerkship phase to observe a broader diversity of competencies [8]. Some early students need guidance in fully developing the critical attributes of trustworthiness, and medical science educators are poised to identify potential deficits and promote student development. This enhances the ability of medical science educators to contribute to learner preparation for entrustment.

Students may not understand how early classroom behaviors contribute to later success in the clinical work place. Describing the educational process as a system in which each student contributes to the learning of others foreshadows the accountability that will be demanded of each student in the health care delivery system. The need for professional, trustworthy behaviors in early educational activities can be framed around preparation for intern duties. This reminder of one's future responsibility for patients may shift student focus from 
Table 3 Sample mapping of Core EPAs to pre-clerkship learning objectives

\begin{tabular}{|c|c|c|c|}
\hline \multirow[t]{2}{*}{ Core EPA } & \multicolumn{3}{|c|}{$\begin{array}{l}\text { Example pre-clerkship learning objectives } \\
\text { Fundamental attributes of trustworthiness required for every EPA }\end{array}$} \\
\hline & EPA-specific knowledge & EPA-specific skills & EPA-specific attitudes \\
\hline $\begin{array}{l}\text { EPA 3: } \\
\text { Recommend and } \\
\text { interpret common } \\
\text { diagnostic and } \\
\text { screening tests }\end{array}$ & $\begin{array}{l}\text { - Defines principles of } \\
\text { screening for disease } \\
\text { - Applies the concepts of } \\
\text { sensitivity and specificity } \\
\text { - Describes the link between } \\
\text { scientific principles and } \\
\text { common diagnostic } \\
\text { measures of } \\
\text { physiological function }\end{array}$ & $\begin{array}{l}\text { - Interprets normal } \\
\text { laboratory values } \\
\text { - Interprets ECGs }\end{array}$ & $\begin{array}{l}\text { Discusses principles } \\
\text { of high-value care when } \\
\text { considering the merits } \\
\text { of various diagnostic and } \\
\text { screening tests }\end{array}$ \\
\hline
\end{tabular}

scores and grades, providing an alternate perspective that facilitates professional identity formation [9].

\section{Medical Science Educators and EPAs in the Clinical Phase of Training}

Clinical judgment and situational awareness are essential to the successful performance of many EPAs. A strong foundational knowledge base is critical. Increasingly, UME programs are devising mechanisms to revisit core scientific concepts later in the curriculum. The Core EPAs offer a framework for potential collaborations between clinical and science educators to enhance vertical integration of core science content throughout all stages of UME training. Referring back to our example in Table 3, the scientific rationale behind commonly used diagnostic texts could be revisited in the context of senior-level clinical rotations. Many clinical services utilize care pathways and protocols; this can create a false impression of a "cookbook" approach. But, those protocols are founded upon scientific evidence of varying degrees of credibility. Medical science educators can be enlisted to help students revisit the scientific rationale. This vertical integration, at a developmental point at which the learner perceives a strong "need to know" and relevance to personal goals, should foster deeper integration of knowledge. It is hoped that the framework of entrustment will encourage learners to focus on truly preparing for their duties as interns. This may stimulate a deeper appreciation for the scientific underpinning of clinical decisionmaking.

Medical science educators are skilled in processes of inquiry. The abilities to recognize a problem, formulate a hypothesis, pursue information, and devise solutions are important to clinical reasoning activities associated with multiple EPAs and are particularly emphasized in EPA 7 ("form clinical questions and retrieve evidence to advance patient care") and EPA 13 ("identify system failures and contribute to a culture of safety and improvement"). Medical science educators may be able to provide insights from the research enterprise and from their experiences educating graduate students to foster and assess these activities in medical students.

\section{Incorporating the Core EPA Framework}

The pilot institutions are currently in the process of introducing curricular interventions and assessments relevant to the EPAs. The group does not expect to render any formal entrustment decisions for several years. First, the pilot members seek to understand what body of performance evidence about each learner will be sufficient to support formal entrustment and will collaborate to address issues of process and feasibility. Current interventions at pilot schools include more deliberate mapping of content and assessments, measures of student trustworthiness, pilots of workplace-based assessments, and faculty and student development in the framework. The challenge of creating structures to support summative entrustment decisions is a current focus of the collective effort, exploring the following: committee structure, composition, and training; longitudinal views of performance, including portfolios; capture of meaningful assessments in a variety of contexts; and development of options for remediation and coaching.

As in any change management process, the incorporation of this novel perspective on teaching and assessing medical students will require collaboration, training, and deliberate oversight. The Faculty Development Concept Group for the Core EPA Pilot emphasizes the need for explicit training regarding the EPA construct for curricular designers, assessors, and students. The Core EPA Pilot will continue to disseminate conceptual viewpoints and practical tools for implementation via presentations at educational conferences, future manuscripts, and online platforms. Educators are encouraged to join the Core EPA list serve for regular literature updates and dynamic discussions (send a blank email to subscribe-coreepas@lists.aamc.org). 
Additional resources are available on the Core EPA website (https://www.aamc.org/initiatives/coreepas/). An understanding of the Core EPAs for Entering Residency will position medical science educators to optimally contribute to the future clinical success of their students.

\section{Summary}

The Core Entrustable Professional Activities for Entering Residency provide our first national description of the expected "product" from undergraduate medical education outside of nationally standardized exams. Although the Core EPAs represent only a portion of the knowledge, skills, and attitudes that medical schools seek to provide their students, they clearly articulate fundamental expectations of incoming interns. Collaboration between clinical and medical science educators will be essential to ensure the readiness of medical school graduates to perform these core tasks in the care of patients.

\section{AAMC Core EPAs for Entering Residency Pilot Team Leaders}

Jonathan Amiel, MD, Columbia University College of Physicians and Surgeons; Patrick Cocks, MD, New York University School of Medicine; Karin Esposito, MD, Florida International University Herbert Wertheim College of Medicine; Michael Green, MD, Yale School of Medicine; Kimberly Lomis, MD, Vanderbilt University School of Medicine; George Mejicano, MD, Oregon Health \& Science University School of Medicine; Michael Ryan, MD, Virginia Commonwealth University School of Medicine; Alexander Stagnaro-Green, MD, University of Illinois College of Medicine; Margaret Uthman, MD, McGovern Medical School at The University of Texas Health Science Center at Houston; and Dianne Wagner, MD, Michigan State University College of Human Medicine.
Acknowledgment The authors thank the Association of American Medical Colleges, Robert Englander, Carol Aschenbrener, Maryellen Gusic, Jan Bull, and all of the members of the Core EPAs for Entering Residency Pilot for their support, inspiration, and contributions to this article.

\section{References}

1. Young JQ, Ranji SR, Wachter RM, Lee CM, Niehaus B, Auerbach AD. "July effect": impact of the academic year-end changeover on patient outcomes: a systematic review. Ann Intern Med. 2011;155 Suppl 5.

2. Association of American Medical Colleges (AAMC). Core entrustable professional activities for entering residency: curriculum developers' guide. AAMC iCollaborative, Washington, DC. 2014. https://www.mededportal.org/icollaborative/resource/887. Accessed 1 Jan 2016.

3. Ten Cate O, Chen HC, Hoff RG, Peters H, Bok H, van der Schaaf M. Curriculum development for the workplace using entrustable professional activities (EPAs): AMEE guide no. 99. Med Teach. 2015;37(11):983-1002. doi:10.3109/0142159 x.2015.1060308.

4. Ten Cate O, Hart D, Ankel F, Busari J, Englander R, Glasgow N, et al. Entrustment decision making in clinical training. academic medicine. 2015(PAP 2015).

5. Core EPA Pilot. Guiding principles for implementing the EPA framework in undergraduate medical education. 2016. https://www.aamc. org/initiatives/coreepas/guiding-principles/. Accessed 11 Feb 2016.

6. Chen HC, van den Broek WES, ten Cate O. The case for use of entrustable professional activities in undergraduate medical education. Acad Med. 2015;90(4):431-6. doi:10.1097 /acm.0000000000000586.

7. Kennedy TJT, Regehr G, Baker GR, Lingard L. Point-of-care assessment of medical trainee competence for independent clinical work. Acad Med. 2008;83(10):S89-92. doi:10.1097 /ACM.0b013e318183c8b7.

8. Burgess AW, McGregor DM, Mellis CM. Applying established guidelines to team-based learning programs in medical schools: a systematic review. Acad Med. 2014;89(4):678-88. doi:10.1097 /acm.0000000000000162.

9. Cruess RL, Cruess SR, Boudreau JD, Snell L, Steinert Y. A schematic representation of the professional identity formation and socialization of medical students and residents: a guide for medical educators. Acad Med. 2015;90(6):718-25. doi:10.1097/acm.0000000000000700. 